\title{
Phytophthora infestans Sporangia Produced in Culture and on Tomato Leaflet Lesions Show Marked Differences in Indirect Germination Rates, Aggressiveness, and Global Transcription Profiles
}

\author{
William E. Fry, ${ }^{1,+}$ Sean P. Patev, ${ }^{1}$ Kevin L. Myers, ${ }^{1}$ Kan Bao, ${ }^{2}$ and Zhangjun Fei ${ }^{2}$ \\ ${ }^{1}$ Section of Plant Pathology and Plant-Microbe Biology, School of Integrative Plant Science, Cornell University, Ithaca, NY, \\ U.S.A.; ${ }^{2}$ Boyce Thompson Institute, Cornell University \\ Accepted 5 November 2018.
}

\begin{abstract}
Sporangia of Phytophthora infestans from pure cultures on agar plates are typically used in lab studies, whereas sporangia from leaflet lesions drive natural infections and epidemics. Multiple assays were performed to determine if sporangia from these two sources are equivalent. Sporangia from plate cultures showed much lower rates of indirect germination and produced much less disease in field and moist-chamber tests. This difference in aggressiveness was observed whether the sporangia had been previously incubated at $4^{\circ} \mathrm{C}$ (to induce indirect germination) or at $21^{\circ} \mathrm{C}$ (to prevent indirect germination). Furthermore, lesions caused by sporangia from plates produced much less sporulation. RNA-Seq analysis revealed that thousands of the $>17,000 P$. infestans genes with a RPKM (reads per kilobase of exon model per million mapped reads) $>1$ were differentially expressed in sporangia obtained from plate cultures of two independent field isolates compared with sporangia of those isolates from leaflet lesions. Among the significant differentially expressed genes (DEGs), putative RxLR effectors were overrepresented, with almost half of the 355 effectors with RPKM >1 being up- or downregulated. DEGs of both isolates include nine flagellar-associated genes, and all were down-regulated in plate sporangia. Ten elicitin genes were also detected as DEGs in both isolates, and nine (including INF1) were up-regulated in plate sporangia. These results corroborate previous observations that sporangia produced from plates and leaflets sometimes yield different experimental results and suggest hypotheses for potential mechanisms. We caution that use of plate sporangia in assays may not always produce results reflective of natural infections and epidemics.
\end{abstract}

Phytophthora infestans is one of the most important and famous of all plant pathogens. It and the diseases it causes (particularly potato and tomato late blight) are the subject of

${ }^{\dagger}$ Corresponding author: W. Fry: wef1@ cornell.edu

Funding: This research was supported by the College of Agriculture and Life Sciences, Cornell University grant number 153-3341.

*The $\boldsymbol{e}$-Xtra logo stands for "electronic extra" and indicates that four supplementary tables are published online.

@ 2019 The American Phytopathological Society several thousand articles annually (Fry 2016). The global costs associated with this pathogen are estimated to be in the range of $\$ 6$ billion annually (Haverkort et al. 2008). Not surprisingly, this pathogen is the subject of intensive investigation at the molecular level (Haas et al. 2009; Kamoun et al. 2015), at the population level (Cooke et al. 2012; Danies et al. 2013; Goodwin et al. 1994), and at the level of practical disease management (Small et al. 2015; Vleeshouwers and Oliver 2013). A common motivation for these studies is to make discoveries that will lead to reductions in the ravages of late blight.

Efficient translation of such discoveries into agricultural application is dependent on assays that are reproducible in both lab and field settings and have sufficient sensitivity to discern quantitative differences. However, assay methods that have proven sufficient to detect the qualitative effects of most resistance $(R)$ genes and many fungicides in past studies may be inadequate to detect the weaker quantitative effects of newer disease controls involving induced and engineered resistance. In fact, there are examples of such resistances being observable in the lab but not in the field (Fry 2016). For example, field studies were unable to confirm the resistance of transgenic potatoes that were overexpressing osmotin (Liu et al. 1994) or the resistance from a pathogen-sensitive promoter that stimulated production of cognate $R$ and avirulence genes (Fry 2016). These failures were surprising, because field assays are capable of accurately detecting small differences in pathogen aggressiveness and host resistance (Fry 1977, 1978; Fry and Apple 1986). Usually such failures are not investigated or are not reported because the failures could be attributed to a plethora of differences between experiments in the lab and the field.

However, recently it has occurred to us that one of these differences, namely the source of sporangia used in an experiment, might be particularly important. This is because a common difference between field and lab experiments is that, in a field experiment (epidemic), there are many cycles of pathogenesis, with the inoculum sporangia coming from leaflet lesions. However, in a lab experiment, the sporangia typically come from a pure culture on an agar medium plate.

Furthermore, we have previously observed two instances in which plate-derived sporangia gave different experimental results than did sporangia from a lesion on a potato or tomato leaflet. The first concerned the sensitivity to solar radiation of sporangia of $P$. infestans; leaflet sporangia survived some exposure to solar radiation but plate sporangia did not (Mizubuti 
et al. 2000). The second concerned induced resistance caused by arachidonic acid (Manosalva et al. 2010). When plate sporangia were used in the bioassay, the lesions on the induced plants were small and often resembled a hypersensitive response, but when leaflet sporangia were used, the lesions on induced plants were not different from those on uninduced plants (Fry 2016).

Additionally, reviewing the literature on inoculation studies with $P$. infestans reveals that the concentration of sporangia in the suspension can range over three orders of magnitude-from hundreds of sporangia per milliliter (Mayton et al. 2010) to hundreds of thousands of sporangia per milliliter (Kirk et al. 2009; Vargas et al. 2009). Often, experiments that use very large sporangial concentrations obtain sporangia from pure cultures growing on agar (Kirk et al. 2009; Tooley and Fry 1985). Presumably, large concentrations are used out of necessity, to achieve sufficient levels of infection.

Because of these suggestions that the quality of inoculum sporangia may be a critical and underappreciated variable in $P$. infestans-plant interaction studies, we chose to systematically compare sporangia from plate cultures and leaflet lesions. Throughout this work, we used a succession of fresh field isolates of $P$. infestans that were all defined as belonging to clonal lineage US-23, which is pathogenic on potato and tomato (Danies et al. 2013), and we addressed three characteristics of the sporangia: i) the rate of indirect germination, ii) the relative ability to cause disease (aggressiveness) in tomato, and iii) global gene expression profiles. We focused on indirect germination (production of zoospores) because it is a basic characteristic of Phytophthora spp. and is commonly assumed to be important to pathogenicity (Judelson and Roberts 2002). Indirect germination is favored at low temperatures $\left(4\right.$ to $\left.10^{\circ} \mathrm{C}\right)$ but is repressed at higher temperatures $\left(20^{\circ} \mathrm{C}\right)$. High temperatures favor direct germination (Danies et al. 2013; Judelson and Roberts 2002; Melhus 1915). Here, we report major differences between sporangia from agar plates and leaflet lesions in indirect germination, aggressiveness, and gene expression profiles.

Table 1. Indirect germination rate of sporangia from pure culture or from lesions on tomato leaflets $(-\mathrm{L})^{\mathrm{a}}$

\begin{tabular}{lccccc}
\hline Treatment & Exp. A & Exp. B & Exp. C & Exp. D & Average \\
\hline 6 dpt (-P) & 2 & 3 & 3 & 3 & 3 \\
12 dpt (-P) & 3 & 10 & 8 & 8 & 7 \\
6 dpi (-L) & 79 & 40 & 68 & 81 & 67 \\
& Exp. E & Exp. F & Exp. G & & \\
7 dpt (-P) & 6 & 2 & 2 & & 3 \\
7 dpi (-L) & 37 & 64 & 52 & & 51 \\
\hline
\end{tabular}

${ }^{a}$ Values show $\%$ indirect germination after incubation at $4{ }^{\circ} \mathrm{C}$ for $120 \mathrm{~min}$. Seven independent experiments (A to $G$ ) were conducted, each on a separate day. Sporangia from pure culture (-P) were obtained at 6,7 , or 12 days posttransfer (dpt). Sporangia from lesions on tomato leaflets (-L) were obtained at 6 or 7 days postinoculation (dpi). Sporangia from pure culture or from leaflets were harvested into a water suspension within 30 min of each other and then incubated at $4^{\circ} \mathrm{C}$ for $120 \mathrm{~min}$. Immediately after harvesting sporangia, an aliquot was assessed for indirect germination at zero time. The average zero time percentage of empty sporangia for suspensions from pure culture were 3,3 , and $9 \%$ for 6,7 , and $12 \mathrm{dpt}$, respectively. The average zero time percentage for suspensions from leaflets were 4 and $2 \%$ for 6 and 7 dpi, respectively. Subsequently, the percent indirect germination after incubation at $4{ }^{\circ} \mathrm{C}$ for $2 \mathrm{~h}$ was determined by counting the number of full and empty sporangia and calculating the percent germinated (no. empty/no. total $\times 100$ ). The percent indirect germination is corrected for the zero time indirect germination. At least 175 sporangia were counted for each of two technical repeats of each experiment. The number reported is the average of these two technical replicates.

\section{RESULTS}

Sporangia from plate cultures show reduced rates of indirect germination and encysted zoospore production relative to leaflet sporangia regardless of sporangia age.

The indirect germination of sporangia from a pure culture on an agar medium plate was compared with that of sporangia from lesions on a tomato leaflet using an isolate of $P$. infestans clonal lineage US-23, as previously defined (Danies et al. 2013). This isolate (LC) had been obtained from a field sample four months before the initiation of the experiments. The agar medium was a 1:1 mixture of pea agar (McLeod et al. 2008) and rye B agar (Caten and Jinks 1968). The tomato cultivar was 'Rutgers'. For each experiment, the sporangia from both sources were produced side by side in the lab at the same time at $21^{\circ} \mathrm{C}$, with a 12 - to 14 -h photoperiod.

The indirect germination rates of plate sporangia were dramatically lower than those of leaflet sporangia (Table 1). Four independent experiments conducted on four different dates compared the indirect germination rates of sporangia from plate culture harvested at 12 days posttransfer (dpt) with that of sporangia from leaflet lesions harvested at 6 days postinoculation (dpi). Under the conditions we used (discussed below), at the time sporangia were harvested, the cultured colony typically had expanded to $90 \%$ of the area of the $9-\mathrm{cm}$ plate and sporulation on the leaflet typically covered most of the leaflet area. In each experiment, the assessments of indirect germination for both types of sporangia occurred within the same 30-min time period, i.e., after sporangia had been harvested into water and had been incubated at $4^{\circ} \mathrm{C}$ for $2 \mathrm{~h}$. Assessments were done microscopically to determine the percentage of sporangia that had released zoospores. For plate sporangia, the indirect germination rate ranged from 3 to $10 \%$, with an average of $7 \%$, and for leaflet sporangia the rate of indirect germination ranged from 40 to $81 \%$, with an average of $67 \%$ (Table 1).

Because it has been reported that 'old' sporangia of Phytophthora spp. (grown in pure culture) lose the ability to release zoospores (Chapman and Vujicic 1965; King et al. 1968), it was important to determine if the indirect germination rate differences between plate sporangia (harvested at $12 \mathrm{dpt}$ ) and leaflet sporangia (harvested at $6 \mathrm{dpi}$ ) were due to differences in the age of sporangia. Therefore, in four independent experiments, we compared the indirect germination rates for sporangia from plate cultures and leaflets harvested at $6 \mathrm{dpt}$ or dpi, respectively, and in another three independent experiments, we compared sporangia harvested from the two sources at $7 \mathrm{dpt}$ or dpi. In each of these experiments, both the transfer to plate culture and the inoculation of the leaflet occurred on the same day, as did the subsequent harvesting and assessment of sporangia (Table 1). For sporangia from plate culture at $6 \mathrm{dpt}$, the indirect germination rate ranged from 2 to $3 \%$, whereas for sporangia from leaflet lesions at $6 \mathrm{dpi}$, the rate ranged from 40 to $81 \%$ with an average of $67 \%$. For sporangia from pure culture at 7 $\mathrm{dpt}$, the indirect germination rate ranged from 2 to $6 \%$ with an average of $3.3 \%$, whereas for sporangia from leaflet lesions, the rate ranged from 37 to $64 \%$ with an average of $51 \%$ (Table 1 ). Clearly, the reduced indirect germination of sporangia from a plate culture was not caused by the age of the sporangia.

Another metric for assessing the indirect germination rate is to determine the number of zoospores per sporangium after the sporangial suspension had been incubated for $3 \mathrm{~h}$ at $4^{\circ} \mathrm{C}$. Because swimming zoospores are extremely difficult to count in a hemacytometer, in this assay, they were stimulated to encyst before counting. The sources of sporangia were isolate LC plate cultures at 6,7 , or $12 \mathrm{dpt}$ or leaflet lesions at 6,7 , or $12 \mathrm{dpi}$. For sporangia from plate cultures, the number of encysted 
zoospores per sporangium ranged from $<0.03$ to 0.5 and, for sporangia from lesions, the cysts per sporangium ranged from 1.94 to 4.00 (Table 2). Importantly, the oldest sporangia from a leaflet lesion (12 dpi) had a high number of zoospores per sporangium (2.91), but the sporangia from pure culture at 12 dpt produced only 0.31 zoospores per sporangium (Table 2 ). In the 12-dpi treatment, the leaflet had been completely colonized by $P$. infestans by day 6 or 7 and there was abundant sporulation over the entire leaflet. It was not apparent that more sporangia were produced in addition to those that were present at 7 dpi. These observations further argue against sporangial age as being responsible for the superior ability of leaflet-derived sporangia to indirectly germinate via the production of zoospores.

\section{Sporangia from plate cultures show reduced indirect germination rates regardless of light regime.}

Because some investigators use sporangia that have been produced in the dark (Tian et al. 2015), it was important to determine if the difference in indirect germination rate between plate sporangia and leaflet sporangia (isolate LC) was due to the light regime. Therefore, in two separate assays, we compared the indirect germination rates of plate sporangia produced in the dark (7 or $10 \mathrm{dpt}$ ) with leaflet sporangia produced in the dark (7 or $10 \mathrm{dpi)}$ (Supplementary Table S1). As a control, we also assessed the indirect germination rates of plate sporangia produced in a 12 - to $14-\mathrm{h}$ photoperiod and leaflet sporangia produced in a 12- to 14 -h photoperiod. In plate sporangia produced in the dark, the indirect germination rate averaged $14 \%$, whereas plate sporangia produced in the $12-$ to 14-h photoperiod had an indirect germination rate that averaged $3 \%$, so there may be an effect of light regime. However, the sporangia produced from leaflets still had much higher indirect germination rates (60 to $65 \%$ ) than did sporangia produced in culture plates (3 to $13 \%$ ). The indirect germination rates of leaflet sporangia produced in the dark (average of 65\%) was not significantly different from that for leaflet sporangia produced in the 12- to 14-h photoperiod (average of 60\%).

\section{Sporangia from plate cultures produce less disease in field and moist-chamber experiments.}

Because we were interested in testing sporangia as commonly prepared for inoculation studies, we compared sporangia from plate cultures at $12 \mathrm{dpt}$ with sporangia from leaflet lesions at 6 dpi. We used the tomato cultivar Jetstar and two $P$. infestans isolates of the US-23 lineage, isolate 112 (obtained from the

Table 2. Numbers of encysted zoospores per sporangium ${ }^{\mathrm{a}}$ in suspensions of sporangia from pure culture or in suspensions of sporangia from lesions on tomato leaflets

\begin{tabular}{lccccc}
\hline Treatment & Exp. A & Exp. B & Exp. C & Exp. D & Average \\
\hline 6 dpt (-P) & 0.07 & $<0.03$ & 0.08 & 0.07 & 0.06 \\
12 dpt (-P) & 0.11 & 0.50 & 0.16 & 0.10 & 0.21 \\
6 dpi (-L) & 3.45 & 1.94 & 2.38 & 3.56 & 2.83 \\
& Exp. E & Exp. F & Exp. G & & \\
7 dpt (-P) & 0.06 & $<0.03$ & 0.2 & & 0.09 \\
7 dpi (-L) & 2.70 & 4.00 & 3.14 & & 3.28 \\
& Exp. H & Exp. I & & & \\
12 dpt (-P) & 0.55 & 0.06 & & & 0.31 \\
12 dpi (-L) & 3.65 & 2.17 & & & 2.91 \\
\hline
\end{tabular}

a The proportion of cysts per sporangium, from nine independent experiments (A to I); assessments for each experiment were conducted on a separate day. Sporangia from pure culture (-P) were obtained at 6,7 , or 12 days posttransfer (dpt). Sporangia from lesions on tomato leaflets (-L) were obtained at 6 or 7 days postinoculation (dpi). Sporangia from pure culture or from leaflets were harvested into a water suspension within 30 min of each other and were then incubated at $4^{\circ} \mathrm{C}$ for $120 \mathrm{~min}$. field in summer 2014) and isolate NV (obtained from the field in summer 2015). For each experiment, the sporangia from both sources had been produced side by side in the lab at the same time, at $21^{\circ} \mathrm{C}$, with a 12 - to 14 -h photo period, and inoculations from both sources were all done in the evening of the same day. Disease severity was assessed visually at $6 \mathrm{dpi}$ with the aid of standard disease diagrams (discussed below). Plants inoculated with sporangia from a plate culture (isolate designation with a '-P' suffix) were much less severely diseased $(P<0.05)$ than plants inoculated with the same number of sporangia from leaflet lesions (isolate designation with a '-L' suffix) (Table 3). For isolate 112, inoculations with sporangia from plate culture produced about $10 \%$ as much disease as inoculations with sporangia from leaflet lesions (Table 3). Isolate 112-P had been passaged at least 12 times on agar medium prior to the experiment and isolate 112-L had been passaged through leaflets at least three times. For isolate NV, the results were in the same direction, but differences were not as large; inoculations with NV-P produced about $30 \%$ as much disease as did inoculations with NV-L (Table 3). Isolate NV-P had been passaged twice on agar medium prior to the field experiment and NV-L had been passed through leaflets three times subsequent to receipt in the lab.

Reduced aggressiveness of sporangia from plate culture (compared with sporangia from a tomato leaflet) was also observed in inoculations done in moist chambers in the lab (Table 3). The amount of disease was assessed visually as above. The moist-chamber experiments were conducted with isolates 112 and $\mathrm{NV}$, as described above, but subsequent to the field experiments. Thus, for the moist-chamber experiments, the sporangia on plate cultures had been passaged on plate culture up to three additional times compared with sporangia used in the field experiments. In each experiment, inoculations from both sources were all done on the same day. Sporangia were produced as described for the field experiment. Disease was assessed at $6 \mathrm{dpi}$. For isolate 112, the differences were even larger than those observed in the field, with sporangia from 112 -P producing $1 \%$ disease and 112 -L producing $87 \%$ disease. For isolate NV the magnitude of difference was similar to that observed in the field, with NV-P sporangia producing $27 \%$ disease and NV-L sporangia producing $80 \%$ disease (Table 3 ). Thus, the use of two $P$. infestans isolates in both field and moistchamber assays and in multiple experimental repetitions all consistently indicate that sporangia from culture plates are markedly less aggressive than those from leaflet lesions.

\section{Sporangia from plate cultures produce less disease, even under conditions favoring direct germination, and those lesions produce fewer sporangia.}

To determine if the differences in aggressiveness observed above were due primarily to differences in indirect germination,

Table 3. Disease severity in field and lab inoculation studies ${ }^{\mathrm{a}}$

\begin{tabular}{lccc}
\hline Treatment & Field Trial 1 & Field Trial 2 & Moist chamber \\
\hline Water & 0 & 0 & 0 \\
$112-\mathrm{P}$ & $5 \mathrm{~b}$ & $1 \mathrm{w}$ & $1 \mathrm{n}$ \\
$112-\mathrm{L}$ & $50 \mathrm{~d}$ & $55 \mathrm{z}$ & $87 \mathrm{p}$ \\
NV-P & $15 \mathrm{c}$ & $10 \mathrm{x}$ & $27 \mathrm{o}$ \\
NV-L & $40 \mathrm{~d}$ & $30 \mathrm{y}$ & $80 \mathrm{p}$ \\
\hline
\end{tabular}

a Values shown as percent disease. Assessments were done at 6 days postinoculation. Plants were inoculated, with the different isolates (112 or $\mathrm{NV}$ ), using sporangia from pure culture plates (-P) or with sporangia from leaflets (-L). Different letters after numbers in a column indicate significant difference $(P<0.05)$. There was no disease in the mockinoculated (water) plants and these zeros were not used in the statistical analysis. 
we assayed sporangia (isolate LC) under conditions that favored direct germination and prevented indirect germination. We harvested sporangia into water at $21^{\circ} \mathrm{C}$ and inoculated leaflets in moist chambers at $21^{\circ} \mathrm{C}$. The number of zoospores produced (cysts per sporangium) was dramatically reduced for sporangia from both sources (Table 4). However, there still tended to be fewer zoospores produced by sporangia from plate cultures than from leaflets (Table 4). Under these conditions, isolate LC-P again produced much less disease than did isolate LC-L (Table 4), with differences similar to those obtained with cold-treated isolates 112 and NV. Thus, there appear to be factors additional to indirect germination rates contributing to the higher aggressiveness of sporangia from leaflet lesions.

In this experiment, we also assessed the number of sporangia produced from the disease lesions that resulted from inoculation with LC-P versus inoculation with LC-L. There were many fewer sporangia produced from the lesions caused by LC-P than from lesions produced by LC-L (Table 4). In one of the six comparisons, sporangia production from lesions produced by LC-P was about $18 \%$ of that in lesions produced by LC-L. In the other five comparisons, sporangial production in lesions caused by LC-P was less than $10 \%$ of that in lesions produced by LC-L (Table 4). Thus, the reduced aggressiveness of sporangia from culture plates goes beyond the production of symptoms to the production of new sporangia that would be needed to drive the next cycle in a field epidemic.

\section{Sporangia from plate cultures and leaflet lesions \\ have markedly different transcriptome profiles.}

The phenotypic differences between plate and leaflet sporangia prompted us to compare global gene expression profiles using RNA-Seq. We compared gene expression in sporangia produced under the particular conditions typically used for inoculation, that is, from 9 -cm culture plates at $12 \mathrm{dpt}$ and from leaflet lesions at $6 \mathrm{dpi}$. Because these experiments were initiated eight months after the conclusion of the disease assessment experiments presented above, we re-evaluated the aggressiveness of the test isolates in new moist-chamber experiments. Two trials of the moist-chamber inoculations were conducted just prior to initiation of the gene-expression studies. At the time of these inoculations (May to June 2016), isolate 112-P had been passaged on agar medium in petri plates for at least 20 transfers, and we found it to no longer produce lesions on tomato within 6 dpi. This is consistent with the observation that isolates passaged in culture for a long time can lose the ability to cause disease (Jinks and Grindle 1963). Consequently, we removed isolate 112 from further consideration. In May to June 2016, NV-P had been passaged on agar medium for at least nine transfers, and NV-L had been passaged through tomato leaflets for three cycles. Isolates NV-P and NV-L were still pathogenic, with NV-P sporangia producing $5 \%$ disease and NV-L sporangia producing $90 \%$ disease in moist chambers. Because of the difficulty with isolate $112-\mathrm{L}$, we examined another isolate of US-23 (GC) that had been obtained in the spring of 2016. Isolate GC-P had been passaged on agar medium for two cycles and GC-L had been maintained consistently on tomato leaflets since receipt of the infected leaflet sample in the lab. Isolates GC-P and GC-L produced 20 and $80 \%$ disease, respectively, in moist chambers. Consequently, the RNA-Seq analysis was done with plate and leaflet sporangia from isolates NV and GC.

Of the 17,785 predicted genes in the $P$. infestans genome (Haas et al. 2009), 15,823 were detected with an RPKM (reads per kilobase of exon model per million mapped reads) $>1$ ) in at least one of the samples (Supplementary Table S2). A summary of the Illumina sequencing data is provided in Table 5. The number of cleaned reads in each sample that were mapped to the $P$. infestans genome ranged from 6.9 million to 15.2 million and the total percentage of reads mapped ranged from 58 to 75 (Table 5). Correlations among the two biological replicates of each treatment ranged from 0.86 to 0.98 .

Table 5. Summary statistics of RNA-Seq data

To Phytophthora infestans

\begin{tabular}{lccrc} 
Isolate & Total raw reads & Cleaned reads & Mapped & \% Mapped \\
\cline { 4 - 5 } GC-L-rep1 & $13,817,156$ & $12,030,115$ & $6,988,040$ & 58.09 \\
GC-L-rep2 & $16,347,753$ & $11,438,658$ & $8,254,638$ & 72.16 \\
GC-P-rep1 & $15,605,635$ & $10,528,066$ & $7,944,789$ & 75.46 \\
GC-P-rep2 & $18,926,343$ & $16,116,506$ & $10,481,630$ & 65.04 \\
NV-L-rep1 & $20,040,006$ & $12,814,905$ & $7,816,326$ & 60.99 \\
NV-L-rep2 & $12,817,104$ & $10,788,956$ & $7,480,226$ & 69.33 \\
NV-P-rep1 & $25,740,953$ & $21,010,466$ & $15,292,136$ & 72.78 \\
NV-P-rep2 & $13,985,409$ & $11,967,468$ & $8,061,983$ & 67.37 \\
\hline
\end{tabular}

Table 4. Disease severity and number of sporangia per leaflet on tomato (cultivar Rutgers) ${ }^{\mathrm{a}}$

\begin{tabular}{|c|c|c|c|c|c|}
\hline \multirow[b]{2}{*}{ Experiment } & \multirow[b]{2}{*}{ Cysts $^{b}$} & \multirow[b]{2}{*}{$\%$ Disease $(\mathrm{dpi})^{\mathrm{c}}$} & \multicolumn{2}{|c|}{ Sporangia per } & \multirow[b]{2}{*}{ Fold change $^{d}$} \\
\hline & & & $\mathrm{cm}^{2}$ of leaflet & $\%$ disease & \\
\hline 1 LC-P 5000 & 0.001 & $90 \%$ (5 dpi) & 3,741 & 4,156 & 0.182 \\
\hline 1 LC-L 5000 & 0.088 & $95 \%$ (5 dpi) & 21,713 & 22,855 & - \\
\hline 1 LC-P 500 & 0.001 & $15 \%$ (5 dpi) & 148 & 986 & 0.064 \\
\hline 1 LC-L 500 & 0.088 & $70 \%(5 \mathrm{dpi})$ & 10,834 & 15,477 & - \\
\hline 2 LC-P 5000 & 0.002 & $75 \%$ (4 dpi) & 856 & 1,141 & 0.032 \\
\hline 2 LC-L 5000 & 0.040 & $85 \%$ (4 dpi) & 30,694 & 36,118 & - \\
\hline 2 LC-P 500 & 0.002 & $10 \%$ (4 dpi) & 95 & 950 & 0.033 \\
\hline 2 LC-L 500 & 0.040 & $35 \%$ (4 dpi) & 10,046 & 28,703 & - \\
\hline 3 LC-P 5000 & 0.002 & $50 \%$ (4 dpi) & 195 & 390 & 0.019 \\
\hline 3 LC-L 5000 & 0.007 & $85 \%$ (4 dpi) & 17,151 & 20,177 & - \\
\hline 3 LC-P 500 & 0.002 & $1 \%$ (4 dpi) & 0 & 0 & 0 \\
\hline 3 LC-L 500 & 0.007 & $15 \%$ (4 dpi) & 2,435 & 16,233 & - \\
\hline
\end{tabular}

a Values shown as percent disease resulting from inoculations using sporangia from plates (LC-P) or sporangia from leaflets (LC-L) under conditions favoring direct germination. Sporangia for inoculations were harvested in water at $21^{\circ} \mathrm{C}$ and were then immediately inoculated onto leaflets in moist chambers and were maintained at $21^{\circ} \mathrm{C}$.

${ }^{\mathrm{b}}$ Cysts per sporangium were determined after the sporangium suspensions had been incubated at $21^{\circ} \mathrm{C}$ for $3 \mathrm{~h}$.

${ }^{c}$ Percent disease was assessed at 5 or 4 days postinoculation (dpi). Sporangia from the leaflets were harvested immediately after disease was assessed. Inoculum contained either 5,000 or 500 sporangia per milliliter.

${ }^{\mathrm{d}}$ Fold change between number of sporangia from lesions LC-P vs. LC-L. 
There were thousands of significant (false discovery rate [FDR] $<0.05$ and fold change $>2$ ) differentially expressed genes (DEGs) in comparisons between plate and leaflet sporangia (Fig. 1; Supplementary Table S3). For the comparison involving the isolate for which there had been only two transfers on agar medium (GC-P vs. GC-L), there were 2,155 DEGs (Fig. 1). For the comparison involving the isolate for which there had been at least nine transfers on agar medium (NV-P vs. NV-L), there were 4,791 DEGs. Using data from both isolates, there was a total of 5,398 unique DEGS, which is $34 \%$ of the 15,823 genes detected in the $P$. infestans genome with an RPKM $>1$. In each isolate, more than $55 \%$ of the DEGs were putative uncharacterized proteins.

For both isolates, comparisons of plate and leaflet sporangia revealed many similarities in the DEG profiles. Of the 2,155 DEGs in isolate GC, 1,549 (approximately 72\%) were also identified as DEGs in isolate NV (Fig. 1). Of the 1,549 DEGs shared by both isolates, 1,544 were DEGs in the same direction (Fig. 1). Further support for the biological significance of the sets of up- and downregulated shared DEGs emerged from identification of the top 300 most significant (statistically) DEGs for each isolate. The vast majority (282) of the 300 most significant DEGs in the GC-P vs. GC-L comparison were also identified as DEGs in the NV-P vs. NV-L comparison, and all 282 were DEGs in the same direction.

Genes differentially expressed in sporangia from plate cultures and leaflet lesions are enriched in several classes associated with indirect germination and pathogenesis.

We next examined the DEGs for the presence of genes associated with indirect germination. Of 24 flagellar-associated genes with an RPKM $>1,14$ were DEGs in one or both isolates. This is a significant $(P$ value of hypergeometric test $=0.0128)$ overrepresentation compared with random genes with RPKM $>1$. Nine of the 14 DEGs were common to both isolates, all being significantly downregulated in plate sporangia. The fold change ranged from 0.03 to 0.31 (Table 6). The reduced expression of flagellar genes in plate sporangia was consistent with our observation that indirect germination was reduced in plate sporangia.

We subsequently investigated all putative RxLR effectors (Supplementary Table S4). Of the 559 RxLR effector genes in the $P$. infestans genome, $355(63 \%)$ were detected with an RPKM $>1$ in at least one sample. Of the 355 , almost half (170) were significantly differentially expressed, which is also a significantly higher proportion than for the entire genome (chisquare statistic $=29.2, P$ value $<0.00001)$. In GC, there were 22 RxLR effectors that were up-regulated and 31 that were downregulated in plate relative to leaflet sporangia. In NV, there were 107 putative RxLR effectors that were significantly upregulated and 45 RxLR effectors that were significantly down-regulated in plate sporangia. Of the 22 that were upregulated in isolate GC-P, 17 were also significantly upregulated in isolate NV-P. Of the 31 that were down-regulated in isolate GC-P, 18 were also significantly down-regulated in isolate NV-P. For these comparisons (plate vs. leaflet), there was no instance in which an RxLR effector was significantly up-regulated in one isolate while significantly down-regulated in the other isolate.

We then refined our analysis of RxLR effectors to focus on those in the top 300 most significant DEGs for each isolate. In isolate GC, there were eight putative RxLR effector genes in the top 300 DEGs, with four downregulated and four upregulated. In isolate $\mathrm{NV}$, there were 13 putative RxLR effectors in the top 300 DEGs, with two downregulated and 11 upregulated. Three effector genes were in the 300 most significant DEGs for both isolates, with two upregulated (PITG_14739 and PITG_14736) and one (PITG_14879) downregulated in plate sporangia compared with leaflet sporangia.

We then investigated the regulation of elicitins in plate and leaflet sporangia, because elicitins have a long history of involvement in the interaction between Phytophthora spp. and their hosts (Fawke et al. 2015). Elicitins (elicitors) are 10-kDa proteins that stimulate an hypersensitive response in some plants. They bind sterols and may be involved in lipid transfer, but their precise role in the physiology of Phytophthora spp. remains somewhat uncertain (Fawke et al. 2015). INF1 is probably the best known elicitin from $P$. infestans and has been shown to be downregulated in planta compared with growth in plate culture on medium (Kamoun et al. 1997). However, INF1 was detected late in infection (Kamoun et al. 1997), particularly in the necrotic part of the lesion (Zuluaga et al. 2016). Of the 41 elicitin genes detected with RPKM $>1,19$ were DEGs, a number that tended to overrepresentation $(P$ value $=0.0705)$. Ten elicitin genes were detected as DEGs in both isolates (Table 7). Nine (including INF1) were up-regulated in plate sporangia compared with leaflet sporangia and one was down-regulated (Table 7).

\section{Analysis of the top 40 DEGs that are most up-regulated or down-regulated reveals additional differences between sporangia from plate cultures and leaflet lesions. \\ We also examined the top 40 most up-regulated DEGs in the GC-P vs. GC-L comparison that were also DEGs in the NV-P}
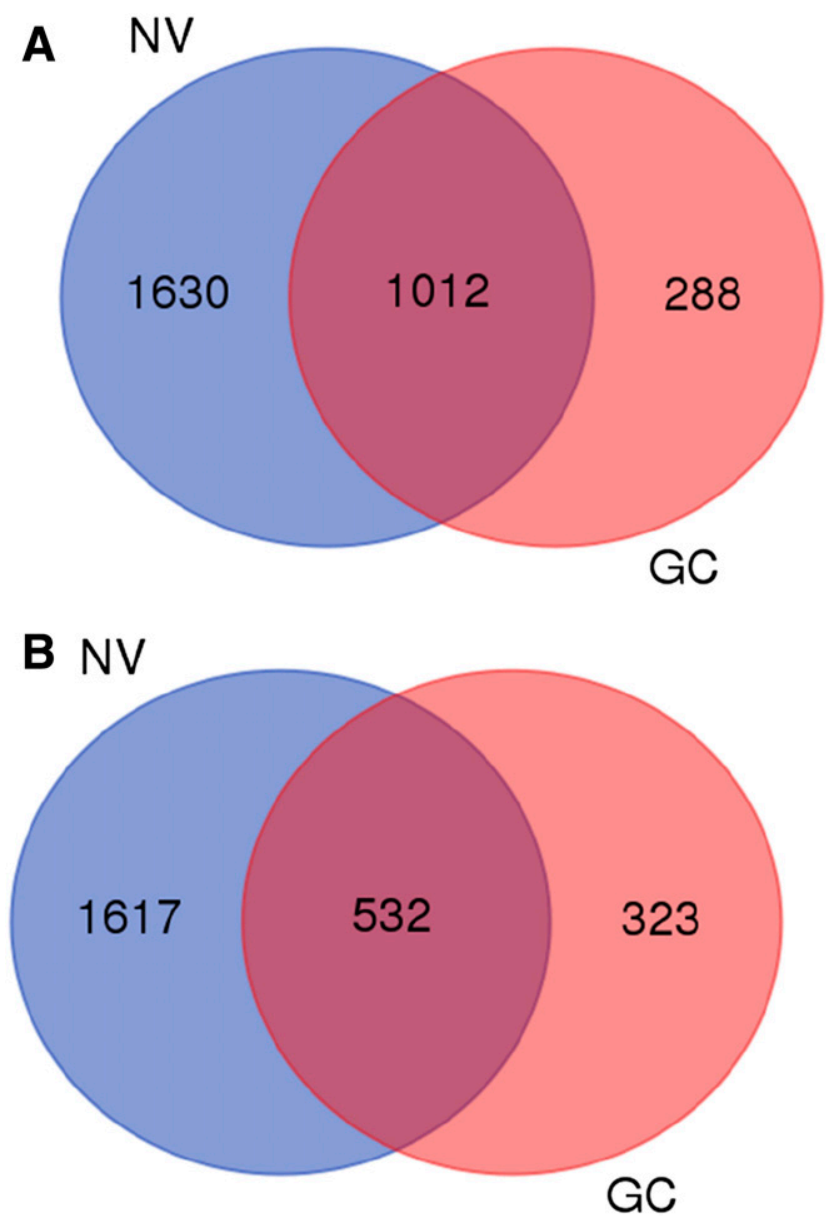

Fig. 1. A Venn diagram illustrating the commonality of significantly differentially expressed genes (DEGs) between sporangia from plates and those from leaves in isolates NV and GC. A, Downregulated genes in the plate sporangia to leaflet sporangia comparison. B, Upregulated genes in the plate sporangia to leaflet sporangia comparison. Five DEGs in one direction in GC were DEGs in the opposite direction in NV. 
vs. NV-L comparison. Most genes were uncharacterized putative proteins, but 17 had probable functions (Table 8), which can be grouped into three broad categories: i) adaptation to growth in a rich medium, ii) response to stress, and iii) other.

Adaptation to a rich medium (six genes). Four genes are thought to be involved in sugar or solute transport. MtN3-like protein is a sugar transporter, and the major facilitator super family proteins (three genes) are thought to be involved in solute transport. Two genes (the arabinogalactan galactosidase and the glucan glucosidase) may be involved in metabolism of components of the medium, which contains plant extracts.

Stress response (four genes). Protein-L-isoaspartate $O$-methyl transferase may repair damaged proteins. Heat-shock 70-kda proteins (two genes) are thought to be involved in a variety of cellular processes including protection of proteins from stress. Nitroreductases reduce NO compounds and they have been suggested to be involved in an oxidative stress response, but their physiological functions are not known with certainty.

Other (seven genes). These upregulated genes are named, but their functions in $P$. infestans cells are not completely known. There were two elicitin-like proteins, a HAM34-like putative membrane protein was detected, and an RxLR effector peptide protein was detected. Some effectors have been shown to interfere with host signaling, but we could not find a precise role of this effector. 12-Oxophytodienoate reductase is involved in the synthesis of jasmonate and, therefore, probably is involved in signaling, particularly in the necrotrophic stage of this hemibiotrophic pathogen. A C2 domain-containing protein is probably targeted to the cell membrane, but the function of the gene upregulated in this study is unknown. A long-chain fattyacid CoA ligase was detected that activates long-chain fatty acids for degradation or for synthesis of cellular lipids.

The 40 DEGs that were most down-regulated in the GC-P vs. GC-L comparison that were also DEGs in the NV-P vs. NV-L comparison were also investigated. Again, most DEGs were uncharacterized putative proteins, but nine had potential functions (Table 9). Two were RxLR effectors, one was a glucoside hydrolase, and the remaining six have likely functions in regulation and signaling. One of these six (nuclear LIM factor interactor-interacting protein-specific sporespecific form) had been previously described as very highly upregulated when sporangia of $P$. infestans produced from a plate culture in agar medium had been exposed to cold to stimulate the release of zoospores (Tani et al. 2004). The strong downregulation of this LIM factor in GC-P is consistent with the reduced ability of sporangia from plate cultures to germinate indirectly.

\section{Expression of Inf-1 as determined by quantitative real-time polymerase chain reaction (q-RT-PCR) corroborates RNA-seq data.}

As an independent control of the RNA-seq data, we assessed, via q-RT-PCR, the expression of elicitin Inf-1 (PITG_12551) in plate and leaflet sporangia. We used actin $\left(P I T G_{-} 14461\right)$ as a normalizer for isolates NV and GC. Inf- 1 was up-regulated in sporangia from plates relative to that in sporangia from leaflets

Table 6. Flagellar-associated significant differentially expressed genes (DEGs) ${ }^{\mathrm{a}}$ in both GC-P vs. GC-L and NV-P vs. NV-L

\begin{tabular}{|c|c|c|c|}
\hline \multirow[b]{2}{*}{ Gene } & \multirow[b]{2}{*}{ Annotation } & \multicolumn{2}{|c|}{ Fold change ${ }^{b}$ in } \\
\hline & & GC-P vs. GC-L & NV-P vs. NV-L \\
\hline PITG_02248 & MBO2, coiled-coil flagellar protein & 0.03 & 0.08 \\
\hline PITG_20295 & RIB43 flagellar protofilament ribbon protein & 0.09 & 0.09 \\
\hline PITG_02163 & RIB43 flagellar protofilament ribbon protein & 0.09 & 0.09 \\
\hline PITG_17132 & Flagella-associated protein & 0.13 & 0.06 \\
\hline PITG_10094 & Intraflagellar transport protein 88 & 0.14 & 0.26 \\
\hline PITG_20212 & Intraflagellar transport protein 172 & 0.14 & 0.31 \\
\hline PITG_18714 & Intraflagellar transport protein 80 & 0.19 & 0.24 \\
\hline PITG_05447 & Sporangia-induced sperm flagellar protein & 0.19 & 0.29 \\
\hline PITG_07309 & Flagellar radial spoke protein & 0.20 & 0.05 \\
\hline
\end{tabular}

${ }^{a}$ DEGs in both comparisons were ranked from greatest to least fold change in the GC-P vs. GC-L.

${ }^{\mathrm{b}} \mathrm{A}$ value of 10 means that the gene was expressed 10-fold greater in the -P sporangia compared with the -L sporangia, a value of 1 means there were no differences in the -P sporangia compared with the $-\mathrm{L}$ sporangia, a value of 0.10 means there was one tenth as much expression in the $-\mathrm{P}$ sporangia as the $-\mathrm{L}$ sporangia.

Table 7. Elicitin or elicitin-like genes that are significantly differentially expressed genes (DEGs) ${ }^{\mathrm{a}}$ in both GC-P vs. GC-L and NV-P vs. NV-L

\begin{tabular}{llrr} 
& & & Fold changeb in \\
\cline { 2 - 4 } Gene & \multicolumn{1}{c}{ Annotation } & GC-P vs. GC-L & NV-P vs. NV-L \\
\hline PITG_04378 & Elicitin-like protein & 66 & 123 \\
PITG_21410 & Elicitin-like INF4 & 63 & 17 \\
PITG_02505 & Elicitin-like protein & 15 & 11 \\
PITG_12562 & Elicitin-like protein & 15 & 15 \\
PITG_16907 & Elicitin-like protein & 12 & 18 \\
PITG_20412 & Putative GPI-anchored serine-rich elicitin & & 11 \\
& SOL13E-like protein & 11 & 146 \\
PITG_12551 & Elicitin (INF1) & 11 & 10 \\
PITG_12556 & Elicitin-like INF6 & 0.14 & 0.08 \\
PITG_12561 & Elicitin INF2A-like protein & & \\
PITG_06908 & Elicitin-like protein &
\end{tabular}

${ }^{a}$ DEGs in both comparisons were ranked from greatest to least fold change in the GC-P vs. GC-L.

${ }^{\mathrm{b}} \mathrm{A}$ value of 10 means that the gene was expressed 10 -fold greater in the $-\mathrm{P}$ sporangia compared with the $-\mathrm{L}$ sporangia, a value of 1 means there were no differences in the $-\mathrm{P}$ sporangia compared with the $-\mathrm{L}$ sporangia, a value of 0.10 means there was one tenth as much expression in the $-\mathrm{P}$ sporangia as the $-\mathrm{L}$ sporangia. 
in both comparisons. For isolate NV, the difference was about fivefold, and, for isolate GC, the difference was about twofold.

\section{DISCUSSION}

Our findings reveal that $P$. infestans sporangia from plate cultures and from leaflet lesions are in distinct physiological states, with a suite of differences that align with their differing abilities to germinate indirectly and to cause disease (Fig. 2). Relative to the more biologically relevant leaflet sporangia, whose primary function is initiating new biotrophic infections, plate sporangia are 'inappropriately' down-regulated in flagellar gene expression and zoospore production but upregulated in elicitins, nutrient transporters, and stress proteins that are more associated with the later, necrotrophic phase of $P$. infestans pathogenesis. Sporangia in this aberrant state are markedly less aggressive regarding lesion development, and subsequent sporulation from those lesions is also markedly reduced. We postulate that signals generated during growth in infected plants but not in plate culture contribute to the transcriptional differences that prime leaflet sporangia for subsequent indirect germination and biotrophic attack. The differential expression of thousands of genes in sporangia from plate cultures compared with those from leaflet lesions is consistent with this model.

All the work presented here involved isolates of the US-23 clonal lineage of $P$. infestans and tests with sporangia from host tomato or rye-pea growth medium. However, because the results with US-23 were so dramatic, we have also initiated preliminary experiments with the US-24 (primarily a potato pathogen) clonal lineage (Danies et al. 2013) and the US-25 clonal lineage (named in summer 2018). These three lineages are genetically distinct from each other. Experiments with these lineages and previous observations with US-1 and US-8 (Mizubuti et al. 2000) are consistent with the data for US-23. For US-24, we found that sporangia from plate culture were again less aggressive than sporangia from leaflet lesions, regardless of whether the culture plates used rye-pea medium or V-8 medium. For US-25, we assessed the indirect germination rates of sporangia produced on tomato leaflet lesions and those of sporangia produced on rye-pea agar medium. The indirect germination rate of US-25 sporangia from the agar medium is dramatically less than that of US-25 sporangia from a leaflet lesion. In one trial, the indirect germination rate of US-25 sporangia from the agar medium averaged just over $2 \%$ and the rate for US-25 sporangia from a leaflet lesion averaged $56 \%$. We have previously reported that for US-1 and US-8, sporangia from an agar medium are very sensitive to solar radiation but sporangia from potato leaflet lesions are somewhat tolerant (Mizubuti et al. 2000). Thus, we have not yet found any exception to our finding that, for $P$. infestans, sporangia from an agar culture are dramatically different from sporangia from a leaflet lesion.

Our results concerning the differences in indirect germination rates of sporangia from agar cultures and from leaflet lesions have precedent in the literature. Melhus (1915) and Danies et al. (2013) obtained sporangia from lesions and reported indirect germination rates of above $70 \%$, whereas Tani and Judelson (2006) obtained sporangia from cultures on ryesucrose agar and reported indirect germination rates of less than $10 \%$.

Our results are also consistent with the results of a recent RNA-Seq analysis using $P$. infestans isolates 1306 (from tomato) and 88069 (from potato) (Ah-Fong et al. 2017). In this very thorough study, the authors compared the gene expression of $P$. infestans grown on rye-sucrose agar in many different stages of development. One of the comparisons was between hyphae prior to sporangiogenesis and sporangia that had been exposed to cold temperature $\left(10^{\circ} \mathrm{C}\right.$ for $\left.60 \mathrm{~min}\right)$ to stimulate zoosporogenesis (cleaved sporangia) (Ah-Fong et al. 2017). In this comparison, all the flagellar-associated genes listed in Table 6 (upregulated in leaflet sporangia compared with plate sporangia) were also up-regulated in cleaved sporangia compared with hyphae. Another of the comparisons conducted by Ah-Fong et al. (2017) was between hyphae prior to sporangiogenesis and sporangia prior to cold treatment. This second comparison identified all except two of the genes in our Table 6 as upregulated in 'uncleaved' sporangia compared with hyphae

Table 8. The 40 most upregulated statistically significant differentially expressed genes (DEGs) ${ }^{\mathrm{a}}$ in the GC-P vs. GC-L comparison that were also statistically significant DEGs in the NV-P vs. NV-L comparison

\begin{tabular}{|c|c|c|c|c|}
\hline \multirow[b]{2}{*}{ Rank in GC } & \multirow[b]{2}{*}{ Gene } & \multirow[b]{2}{*}{ Annotation } & \multicolumn{2}{|c|}{ Fold-change $^{b}$ in } \\
\hline & & & GC-P vs. GC-L & NV-P vs. NV-L \\
\hline 3 & PITG_12872 & MtN3-like protein & $>1,000$ & 40 \\
\hline 10 & PITG_09907 & $\begin{array}{l}\text { Arabinogalactan endo-1,4-beta- } \\
\text { galactosidase }\end{array}$ & $>200$ & 25 \\
\hline 11 & PITG_13006 & Major facilitator superfamily (MFS) & 196 & 52 \\
\hline 14 & PITG_14993 & Protein-L-isoaspartate $O$-methyltransferase & 161 & 8 \\
\hline 18 & PITG_14721 & 12-Oxophytodienoate reductase & 117 & 84 \\
\hline 20 & PITG_09454 & Nitroreductase family & 98 & 12 \\
\hline 22 & PITG_20027 & MFS & 80 & 12 \\
\hline 24 & PITG_09799 & Glucan $1,3-\beta$-glucosidase & 78 & 29 \\
\hline 27 & PITG_03036 & MFS & 77 & 11 \\
\hline 28 & PITG_11655 & Long-chain fatty-acid CoA ligase & 72 & 53 \\
\hline 29 & PITG_04386 & Ham34-like putative membrane protein & 71 & 65 \\
\hline 30 & PITG_11247 & Heat-shock $70-\mathrm{kDa}$ protein & 71 & 26 \\
\hline 32 & PITG_10339 & Secreted RxLR effector peptide protein & 66 & 16 \\
\hline 33 & PITG_04378 & Elicitin-like protein & 66 & 99 \\
\hline 34 & PITG_11853 & $\mathrm{C} 2$ domain-containing protein & 64 & 31 \\
\hline 35 & PITG_21410 & Elicitin-like protein INF4 & 64 & 123 \\
\hline 39 & PITG_11249 & Heat-shock 70-kDa protein & 57 & 21 \\
\hline
\end{tabular}

${ }^{\text {a }}$ DEGs in both comparisons were ranked from greatest to least fold-change in the GC-P vs. GC-L. Most genes (not listed) were putative uncharacterized proteins. Shown here are genes with a putative function.

b A value of 10 means that the gene was expressed 10-fold greater in the -P sporangia compared with the -L sporangia, a value of 1 means there were no differences in the $-\mathrm{P}$ sporangia compared with the $-\mathrm{L}$ sporangia, a value of 0.10 means there was one tenth as much expression in the $-\mathrm{P}$ sporangia as the $-\mathrm{L}$ sporangia. 
on a plate (Ah-Fong et al. 2017). These findings further confirm that sporangia from leaflets (compared with sporangia from plates) are primed for indirect germination.

That the expression profile of PITG_11241 was similar to that of many flagellar genes is also notable in this context. This gene was markedly up-regulated in leaflet sporangia compared with plate sporangia (Table 9). The gene encodes the sporespecific form of a nuclear LIM factor interactor that has a role in indirect germination (Tani and Judelson 2006; Tani et al. 2005) and is up-regulated in plate cultures of $P$. infestans in both the transition from hyphae to sporangia and upon chilling of sporangia that induces cleavage into zoospores (Ah-Fong et al. 2017). Thus, the expression profile of this gene in the developmental stages of $P$. infestans is again consistent with our observation that sporangia from leaflets are primed for indirect germination.

It may be that the differences we have observed between sporangia from plate cultures and sporangia from leaflet lesions are functionally related to the 'degeneration' of oomycete or fungal plant pathogens that have been passaged many times in plate culture (Butt et al. 2006). Certainly, it has been known for decades that isolates of $P$. infestans passaged in plate culture for years can lose the ability to cause disease (Jinks and Grindle 1963). As a result many investigators routinely 're-isolate' $P$. infestans from plants to rejuvenate cultures. We did not observe any morphological differences in sporangia from plates compared with sporangia from leaflets, but, for some fungi, morphological changes have been reported after continued passaging in plate culture (Butt et al. 2006). For Botrytis cinerea, DNA demethylation is thought to play a role in the degeneration (Breen et al. 2017). A major difference between previous observations of degeneration and our study with $P$. infestans is that the differences we document occurred after only a few passages on agar medium and they are reversible by inoculating and re-isolating $P$. infestans from an infected leaflet. The fact that there were more DEGs in the comparison that had experienced the greater number of passages on medium in plate culture (NV) is consistent with a progressive process, possibly involving epigenetic changes. These changes could be involved in degeneration.

However, our finding that leaflet-derived sporangia are better primed for both indirect germination and enhanced aggressiveness, as indicated by bioassays and a functionally coherent set of DEGs, raises an intriguing possible explanation that transcends the degeneration of cultured pathogens. That is, the pathogenic priming of sporangia may be 'tuned' by the infection producing them for ecological benefit to the pathogen and the sporangia from plate cultures represent an extreme in the tunable gene expression spectrum. This hypothesis would be consistent with the host-dependent effects observed with Phytophthora ramorum (Kasuga et al. 2012). P. ramorum isolates of the same clonal lineage have different phenotypic characteristics dependent on whether the oomycete is isolated from oak or bay laurel (Kasuga et al. 2012). The pathogen isolated from oak grew more poorly after subculturing and was more likely to die in long-term storage than was the pathogen isolated from bay laurel (Kasuga et al. 2012). The authors proposed that transposon derepression in the isolates obtained from oak led to these differences. In contrast to the situation demonstrated in our study with $P$. infestans, the observed changed phenotypes in $P$. ramorum were not reported to be reversible. Several of the DEGs identified in our study could be used to test the hypothesis that $P$. infestans can be tuned to a particular host or substrate.

A corollary to this hypothesis of 'tunable priming' of sporangia is that the regulatory status of various DEGs may provide useful clues to their roles in pathogenesis. According to this model, RxLR PITG_10339, which is among the 40 most upregulated DEGs in plate sporangia, may have a role in pathogenesis that occurs later than that of PITG_14360 or PITG_04148, which are among the 40 most downregulated DEGs in plate sporangia. This is based on the assumption that there is biological significance to the 'inappropriate' expression of these RxLR genes in plate-derived sporangia. Importantly, a better understanding of what factors are determinative in pathogenesis, including those controlling the possible priming of developing sporangia, may provide new targets for disease control.

Our experiments have implications for a universal technical issue in the study of culturable plant pathogenic bacteria, fungi, and oomycetes. As a key to Koch's postulates, the use of pure cultures is foundational to the study of a pathogen, and the experimental advantages of using lab-maintained model strains that have a known genotype and whose propagules can be conveniently prepared on defined media typically outweigh concerns that inocula produced in culture may not be physiologically identical to inocula produced in planta. However, our findings also raise a cautionary note. Use of only plate sporangia might lead to false positives in some experiments. For example, resistance that is promisingly able to reduce disease

Table 9. The 40 most downregulated statistically significant differentially expressed genes (DEGs) ${ }^{\mathrm{a}}$ in the GC-P vs. GC-L comparison that were also statistically significant DEGs in the NV-P vs. NV-L comparison

\begin{tabular}{|c|c|c|c|c|}
\hline \multirow[b]{2}{*}{ Rank in GC } & \multirow[b]{2}{*}{ Gene } & \multirow[b]{2}{*}{ Annotation } & \multicolumn{2}{|c|}{ Fold-change $^{\mathrm{b}}$ in } \\
\hline & & & GC-P vs. GC-L & NV-P vs. NV-L \\
\hline 3 & PITG_04148 & Secreted RxLR effector peptide protein & 0.00 & 0.01 \\
\hline 8 & PITG_21079 & RGS-like protein kinase & 0.00 & 0.03 \\
\hline 12 & PITG_06327 & Chromodomain protein & 0.00 & 0.00 \\
\hline 18 & PITG_14360 & Secreted RxLR effector peptide protein & $<0.01$ & 0.06 \\
\hline 19 & PITG_11987 & Glycoside hydrolase & $<0.01$ & 0.02 \\
\hline 23 & PITG_11241 & $\begin{array}{l}\text { Nuclear LIM factor interactor-interacting } \\
\text { protein spore-specific form }\end{array}$ & $<0.01$ & 0.04 \\
\hline 29 & PITG_18578 & Dual specificity protein phosphatase & $<0.01$ & 0.31 \\
\hline 36 & PITG_17951 & $\begin{array}{l}\text { Dicarboxylate/amino acid:cation }(\mathrm{Na} \text { or } \mathrm{H}) \\
\text { symporter (DAACS) family }\end{array}$ & $<0.01$ & 0.06 \\
\hline 38 & PITG_07549 & Protein kinase & $<0.01$ & 0.04 \\
\hline
\end{tabular}

${ }^{a}$ DEGs in both comparisons were ranked from greatest to least fold change in the GC-P vs. GC-L comparison. Most genes (not listed) were putative uncharacterized proteins. Shown here are genes with a putative function.

b A value of 10 means that the gene was expressed 10-fold greater in the -P sporangia compared with the -L sporangia, a value of 1 means there were no differences in the -P sporangia compared with the $-\mathrm{L}$ sporangia, a value of 0.10 means there was one tenth as much expression in the $-\mathrm{P}$ sporangia as the $-\mathrm{L}$ sporangia. 
caused by plate sporangia in lab tests may be ineffective against the more fit and aggressive sporangia generated from disease lesions in the field. On the pathogen side, investigation of a protein that is up-regulated in plate sporangia may lead to incorrect conclusions about the role of that protein in pathogenesis that occurs under natural or field conditions. Additionally, assessment of ecological effects on sporangia from plates might identify a response not shared by sporangia produced in an epidemic.

Importantly, our work reveals a defined set of phenotypic and gene expression differences that can guide more efficient assessment of sporangia fitness. Our findings may also provide clues to the roles of key $P$. infestans proteins in stages in pathogenesis and in survival.

\section{MATERIALS AND METHODS}

\section{$P$. infestans isolates.}

Four different isolates of the US-23 lineage of $P$. infestans were used. Lineage determinations were done in our lab using the criteria described by Danies et al. (2013). Each isolate had been obtained from the field (from a leaf with a single lesion) less than two years before analysis. Isolate 112 was isolated from diseased commercial tomato plants gathered in the state of New York in 2014. Isolate NV was isolated from diseased tomato plants in New York state in the early summer of 2015. Isolate GC was cultured from diseased tomato plants submitted from Florida to our lab for analysis in spring 2016. Isolate LC was obtained from a sample from New York state submitted to our lab in 2017.

After identifying each isolate as clonal lineage US-23 with microsatellites (Danies et al. 2013), it was maintained in the lab at $21^{\circ} \mathrm{C}$ and with a photoperiod of $12-$ to $14-\mathrm{h}$. In plate culture, it was maintained on agar medium in $9-\mathrm{cm}$ petri plates containing a mixture of $1 / 2$ pea (McLeod et al. 2008) and $1 / 2$ rye $\mathrm{B}$ agar (Caten and Jinks 1968). To move an isolate to live tissue from plate culture, sporangia were washed from the plate culture and drops (each $200 \mu \mathrm{l})$ of this suspension $\left(1 \times 10^{3}\right.$ sporangia per milliliter) were deposited on the underside of tomato leaflets in petri-plate moisture chambers (inverted $9-\mathrm{cm}$ petri plates with $20 \mathrm{ml}$ of $1.5 \%$ water agar in the smaller half now on the top [Zuluaga et al. 2016]). Subsequent transfers from leaflet to leaflet were carried out by touching healthy leaflets to diseased sporulating ones and placing the inoculated leaflets in a new water-agar moist-chamber. Agar cultures were transferred by cutting and moving colonized agar cubes from the growing edge of an old culture to a fresh pea and rye agar plate. Under the conditions described here, the pathogen covered about $90 \%$ of the plate by $12 \mathrm{dpt}$. Sporangia from 8- to 12-day-old cultures are commonly used in diverse experiments with $P$. infestans (Ah-Fong et al. 2017; Judelson and Roberts 2002) Transfers onto fresh pea and rye agar plates were typically done every three weeks. Transfers from a diseased leaflet to a healthy one were done typically every 7 to 10 days. In preparation for inoculations, isolates were cultured on a mixture of pea agar and rye $B$ agar or on leaflets of the susceptible tomato cultivars JetStar or Rutgers. Except where described otherwise, pure cultures in agar medium and inoculated or infected leaflets were maintained in the lab at $21^{\circ} \mathrm{C}$ with a 12 - to $14-\mathrm{h}$ photo period. Cultures or inoculated leaflets, in the dark, were wrapped in foil and were maintained immediately after transfer or after inoculation in a closed cabinet at $21^{\circ} \mathrm{C}$.

\section{Indirect germination assays.}

To assess indirect germination, sporangia (from plate culture or from lesions on leaflets) were harvested into water by rubbing the surface of the agar or agitating the leaflet in $30 \mathrm{ml}$ of water in a 50-ml falcon tube. This resulted in a suspension of

\section{Sporangia source}

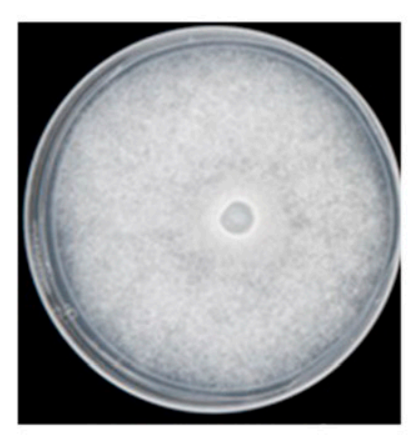

\section{Pathogenicity Elicitins}

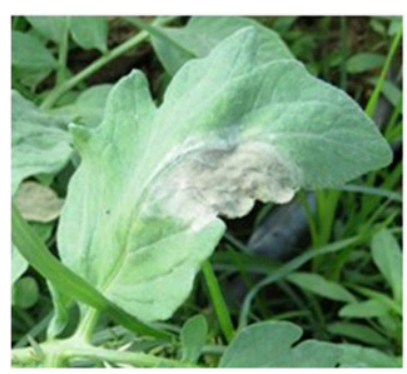

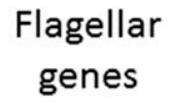
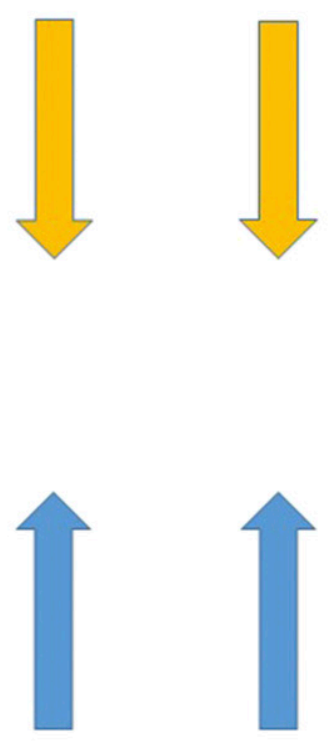

$\begin{array}{cc}\text { Indirect } & \text { Flagellar } \\ \text { germination } & \text { genes }\end{array}$

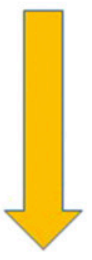

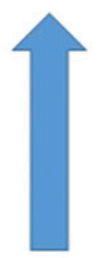

Stress

response

Fig. 2. Summary of differences between sporangia from culture and from tomato leaflets. 
about 20,000 to 30,000 sporangia per milliliter. In each experiment, sporangia from cultures and leaflets were harvested within the same 30-min period. An aliquot was taken for a zerotime assessment (using a hemacytometer) of nongerminated (full) sporangia and of sporangia that had already released zoospores (empty sporangia). The suspension ( 20 to $30 \mathrm{ml}$ ) was then placed at $4^{\circ} \mathrm{C}$ for $2 \mathrm{~h}$, after which time, the numbers of empty and full sporangia were counted, using a hemacytometer. The zero-time proportion was subtracted from the proportion obtained after the 2 -h incubation period. There were at least two technical replications from each suspension, and each technical replication was assessed twice. A minimum of 175 total sporangia was counted in each assessment, except for the experiments involving incubation in the dark, in which at least 75 sporangia were counted for each treatment.

The number of 'cysts per sporangium' was also assessed. It is based on the fact that zoospores encyst when agitated and when contacting a solid surface (Tokunaga and Bartnicki-Garcia 1971). Sporangia were harvested and the initial concentration of sporangia was assessed as described above. A 20-ml aliquot of the suspension was incubated for three $\mathrm{h}$ in a 50-ml Falcon tube at $4^{\circ} \mathrm{C}$, after which time the Falcon tube was inverted several times. Two 1-ml aliquots of the sporangia/zoospore suspension were then transferred to $1.5-\mathrm{ml}$ microfuge tubes. Each tube was vortexed for $40 \mathrm{~s}$ to induce cyst formation, thereby immobilizing zoospores. Cyst concentration (number of cysts per milliliter) in each tube was determined microscopically, using a hemacytometer. The number of cysts per sporangium was then calculated.

\section{Field experiments.}

There were two trials of the field experiment. Trial 1 consisted of two randomized complete blocks and trial 2 consisted of three randomized complete blocks. The susceptible tomato cultivar Jet Star was used in both trials. Seedlings were grown in the greenhouse until they were about 5 inches in height. These plants were hardened off in cold frames for 1 week and were then transplanted to the field at the Thompson research farm in Freeville, NY. The first trial was transplanted on July 10, 2015, and the second trial was transplanted on July 16, 2015. Each treatment was applied to a plot of three plants. Each plot was surrounded by fallow soil for a distance of $3 \mathrm{~m}$.

Treatments consisted of applications of water or a water suspension of $P$. infestans sporangia. Water or sporangial suspensions (discussed below) were applied using Preval 100-ml aerosol sprayers. Each plot received $5 \mathrm{ml}$ of the appropriate preparation to uniformly cover all foliage of these small plants. Each sporangium suspension was adjusted to 5,000 sporangia per milliliter, using a hemacytometer. Each sprayer was used only for a single treatment. Treatments were applied at dusk on August 11, 2015 for trial 1 and at dusk on August 14, 2015 for trial 2. Prior to treatment applications, plots were irrigated with overhead sprinkler irrigation to wet the foliage.

There were five types of treatments in each block, one water treatment (mock inoculation) and four sporangial preparations. The sporangial treatments were 112-P, 112-L, NV-P, and NV-L (as described above). Sporangia from plate cultures were harvested when the isolate had covered about 80 to $90 \%$ of the surface of the pea and rye agar (10 to 12 days after starting the culture). Sporangia were washed off with distilled water, and the concentration in this initial suspension was determined using a hemacytometer, then, adjusted to 5,000 per milliliter. Subsequently, the suspension was placed at $4{ }^{\circ} \mathrm{C}$ for $30 \mathrm{~min}$ to promote zoospore release and was then reassessed, using the hemacytometer to confirm concentration and germination. Sporangial suspensions from leaflet lesions were prepared by washing two tomato leaflets (on which sporulation occurred on approximately $90 \%$ of the leaflet surface) in distilled watertypically at $6 \mathrm{dpi}$. The resulting suspension was then adjusted to 5,000 per milliliter and was incubated at $4{ }^{\circ} \mathrm{C}$, as described above. For each trial of each experiment, all inoculations for all treatments occurred on the same day and all sporangial suspensions were prepared on the day of inoculation.

\section{Isolate treatment prior to inoculation of the field experiments.}

For the first field experiment, 112-P had been passaged on agar medium at least 12 times and 112-L had been passed through leaflets three times. Also, for the first field experiment, NV-P had been transferred twice on pea and rye agar and NV-L had been transferred three times onto leaflets since isolation. For the second field experiment, each isolate had been transferred an additional time on its respective substrate.

\section{Disease assessment.}

One plant from each treatment in each replication was uprooted and placed into a plastic bag at $84 \mathrm{~h}$ postinoculation (hpi) and was kept at $21^{\circ} \mathrm{C}$ until observation at 6 dpi. Bagging plants at 84 hpi prevented sporulation in the field, eliminating the risk of contaminating other experiments or serving as an inoculum source for neighboring commercial growers. At 6 dpi, plants were removed from their bags, were photographed, and were assessed for disease, using a key published by James (1971). This key standardizes disease measurement as coverage $(\%)$ of the foliar surfaces in sporulating lesions. For statistical analysis, we used the general linear model (Proc GLM) in the Statistical Analysis System (SAS 9.3; Cary, NC, U.S.A.). The water controls ( $0 \%$ disease) were eliminated from the comparison and the analyses were performed on percent disease.

\section{Moist-chamber experiments.}

The treatments applied in the field experiment (water, 112-P, 112-L, NV-P, and NV-L) were also applied to detached leaflets in experiments conducted in moist chambers in the lab. The moist-chamber experiments occurred in August to September 2015, subsequent to the field experiments. Leaflets were obtained from tomato plants ('JetStar') growing in the greenhouse. The leaflets were detached from fully developed upper leaves of plants 6 to 8 weeks from seeding. The moist chambers consisted of $15-\mathrm{cm}$ petri plates containing $20 \mathrm{ml}$ of $1.5 \%$ water agar. The plates were inverted (water agar on top in the smaller half) and four or five tomato leaflets were placed in the bottom (larger half). There were three independent trials of the moistchamber experiment with one moist chamber per treatment in each trial. The trials were initiated on the evenings of August 17, September 8, and September 22, 2015.

For the first trial of the moist-chamber experiment, 112-L had been passed through leaflets four times and 112-P had been passaged on agar medium at least 13 times. Also, for the first trial of the moist-chamber experiment, NV-L had been transferred four times onto leaflets since isolation and NV-P had been transferred three times on pea and rye agar. For the second and third trials of the moist-chamber experiment, each isolate had been transferred one additional time on its appropriate substrate for each additional trial.

Water or sporangial suspensions $(5,000$ sporangia per milliliter, except for a few experiments that used 500 sporangia per milliliter, as indicated above) were applied to plant surfaces at the rate of $1.5 \mathrm{ml}$ per moist chamber, using Preval 100-ml aerosol sprayers. Four independent sporangial suspensions for use in inoculation (as described above) were prepared for each trial of the moist-chamber experiment. After the leaflets were sprayed, the chambers were sealed with parafilm to maintain humidity and promote good infection conditions. The moist 
chambers were incubated at $21^{\circ} \mathrm{C}$ with a 12 - to 14 -h photoperiod after treatment. Except for the experiments done at $21^{\circ} \mathrm{C}$, sporangial suspensions were incubated at $4^{\circ} \mathrm{C}$ for $2 \mathrm{~h}$ before inoculation. One or two leaflets per treatment in each trial were selected at random for disease assessment at $6 \mathrm{dpi}$. Again, assessments were guided by using a key published by James (1971). Statistical analyses were performed as described above for the field data.

\section{Sporangia sample preparation for RNA-Seq analysis.}

These experiments were initiated in May to June 2016. Sporangia were prepared for isolates 112-P, 112-L, NV-P, NVL, GC-P, and GC-L. The sources of sporangia were as described for the inoculum preparation. However, suspensions from each source were concentrated by centrifugation $(4 \mathrm{~min}$ at $14,000 \mathrm{rpm}$ ) in 2-ml snap-top tubes to produce pellets of sporangia. Sufficient numbers of petri plates and infected leaflets were used to obtain 300,000 sporangia of each of 112-P, NV-P, and NV-L (smaller numbers of 112-L were obtained as described above). After decanting the supernatant, the pellets were frozen in liquid nitrogen and were then stored at $-80^{\circ} \mathrm{C}$, until further processing. There were two independent suspensions from a plate culture and two independent suspensions from leaflet lesions for two biological replicates of each treatment.

\section{RNA-Seq analysis.}

Frozen pellets of sporangia were ground in extraction buffer (Lexogen Split RNA "IB") using ceramic bead homogenization tubes. RNA was extracted using the Lexogen Split RNA kit. Extractions resulting in more than $100 \mathrm{ng}$ RNA per microliter with absorption characteristics of $260 / 280$ above 2.0 and 260/230 above 1.8, as assessed on a Thermo Scientific NanoDrop were frozen at $-80^{\circ} \mathrm{C}$ for future use.

RNA-Seq libraries were then constructed from total RNA, using the Lexogen SENSE mRNA library kit. This kit excluded ribosomal RNA, using magnetic beads, which associated with poly-A tails found only on mRNA. This allowed ribosomal RNA to be washed away, maximizing coverage of expressed genes when the resulting libraries were sequenced. RT-PCR was used to establish accurate cycling for barcode incorporation and final library amplification, avoiding over- or under-cycling libraries that could skew transcript counts during sequencing. Library fragment size was assessed by fragment analysis, and with concentration data from Qubit analysis of each library, a 2-nM equimolar pool was prepared for each lane of Illumina HiSeq 4000.

Raw RNA-Seq reads were processed using Trimmomatic (Bolger et al. 2014) to remove adaptor and low-quality sequences. Reads shorter than $40 \mathrm{bp}$ after trimming were discarded. RNA-Seq reads were then aligned to the ribosomal RNA database (Quast et al. 2013), using Bowtie (Langmead et al. 2009), and the mappable reads were discarded. The resulting high-quality cleaned reads were aligned to the P. infestans genome (Haas et al. 2009), using HISAT (Kim et al. 2015) allowing up to four mismatches. Following alignments, raw counts for each $P$. infestans gene were derived and normalized to RPKM. The raw counts were also fed to the edgeR program (Robinson et al. 2010) to identify DEGs. Raw $P$ values of multiple testing were corrected using FDR (Benjamini and Hochberg 1995). Genes with a FDR less than 0.05 and fold change $>2$ were identified as DEGs. Annotations of the predicted $P$. infestans genes were obtained from the EnsembleProtists website.

\section{q-RT-PCR.}

Total extracted RNA was transcribed to cDNA using the Applied Biosystems cDNA synthesis kit for reverse transcription.
RT-PCR was carried out on an AbiPrism 7000 and calculation of relative expression was carried out following the protocols outlined by Pfaffl (2004). Primers for INF-1 were forward, 5' GTGGCGCTCGTAAGCATCCT-3', and reverse, 5'-CATA GCGACGCACACGTAGA-3'. Primers for actin were forward, 5'-AGCTGTGGGCACACTTAAT-3', and reverse, 5'-CTGG TCCATGCTCTCATTCA-3'.

\section{ACKNOWLEDGMENTS}

We thank H. Mayton for assistance with the statistical analyses, P. Zuluaga for a very helpful discussion that stimulated this experimental approach, and A. Collmer for his helpful discussion of the findings and for insightful wording suggestions. We also thank two anonymous reviewers of an earlier version of the manuscript, whose (sometimes doubting) comments were very helpful to the development of a more coherent and complete manuscript.

\section{LITERATURE CITED}

Ah-Fong, A. M. V., Kim, K. S., and Judelson, H. S. 2017. RNA-seq of life stages of the oomycete Phytophthora infestans reveals dynamic changes in metabolic, signal transduction, and pathogenesis genes and a major role for calcium signaling in development. BMC Genomics 18:198-219.

Benjamini, Y., and Hochberg, Y. 1995. Controlling the false discovery rate: A practical and powerful approach to multiple testing. J. Roy. Stat. Soc. B Met. 57:289-300.

Bolger, A. M., Lohse, M., and Usadel, B. 2014. Trimmomatic, a flexible trimmer for Illumnina sequence data. Bioinformatics 30:2114-2120.

Breen, J., Mur, L. A. J., Sivakumaran, A., Akinyemi, A., Wilkinson, M. J., and Rodriguez-Lopez, C. M. 2017. DNA methylation plays a role on invitro culture induced loss of virulence in Botrytis cinerea. Published online. Cold Spring Harbor Laboratory, Cold Spring Harbor, NY, U.S.A. http://dx.doi.org/10.1101/059477

Butt, T., Wang, C., Shah, F., and Hall, R. 2006. Degeneration of Entomogenous Fungi. Pages 213-226 in: An Ecological and Societal Approach to Biological Control. J. Eilenberg, and H. M. T. Hokkanen, eds. Springer, Dordrecht, The Netherlands.

Caten, C. E., and Jinks, J. L. 1968. Spontaneous variability of single isolates of Phytophthora infestans. I. Cultural variation. Can. J. Bot. 46:329-348.

Chapman, J. A., and Vujicic, R. 1965. The fine structure of sporangia of Phytophthora erythroseptica Pethyb. J. Gen. Microbiol. 41:275-282.

Cooke, D. E. L., Cano, L. M., Raffaele, S., Bain, R. A., Cooke, L. R., Etherington, G. J., Deahl, K., Farrer, R. A., Gilroy, E. M., Goss, E. M. Grunwald, N. J., Hein, I., MacLean, D., McNicol, J. W., Randall, E., Oliva, R. F., Pel, M. A., Shaw, D. S., Squires, J. N., and Taylor, M. C., Vleeshouwers, V. G. A. A., Birch, P. R., Lees, A. K., and Kamoun, S. 2012. Genome analyses of an aggressive and invasive lineage of the Irish potato famine pathogen. PLoS Pathog. 8:e1002940.

Danies, G., Small, I. M., Myers, K., Childers, R., and Fry, W. E. 2013. Phenotypic characterization of recent clonal lineages of Phytophthora infestans in the United States. Plant Dis. 97:873-881.

Fawke, S., Doumane, M., and Schornack, S. 2015. Oomycete interactions with plants: Infection strategies and resistance principles. Microbiol. Mol. Biol. Rev. 79:263-280.

Fry, W. E. 1977. Integrated control of potato late blight-Effects of polygenic resistance and techniques of timing fungicide applications. Phytopathology 67:415-420.

Fry, W. E. 1978. Quantification of general resistance of potato cultivars and fungicide effects for integrated control of late blight. Phytopathology 68 1650-1655.

Fry, W. E. 2016. Phytophthora infestans: New tools (and old ones) lead to new understanding and precision management. Annu. Rev. Phytopathol. 54:529-547.

Fry, W. E., and Apple, A. E. 1986. Disease management implications of age-related changes in susceptibility of potato foliage to Phytophthora infestans. Am. Potato J. 63:47-56.

Goodwin, S. B., Cohen, B. A., and Fry, W. E. 1994. Panglobal distribution of a single clonal lineage of the Irish potato famine fungus. Proc. Natl Acad. Sci. U.S.A. 91:11591-11595.

Haas, B. J., Kamoun, S., Zody, M. C., Jiang, R. H. Y., Handsaker, R. E., Cano, L. M., Grabherr, M., Kodira, C. D., Raffaele, S., Torto-Alalibo, T., Bozkurt, T. O., Ah-Fong, A. M. V., Alvarado, L., Anderson, V. L., Armstrong, M. R., Avrova, A., Baxter, L., Beynon, J., Boevink, P. C., Bollmann, S. R., Bos, J. I. B., Bulone, V., Cai, G., Cakir, C., Carrington, J. C., Chawner, M., Conti, L., Costanzo, S., Ewan, R., Fahlgren, N., 
Fischbach, M. A., Fugelstad, J., Gilroy, E. M., Gnerre, S., Green, P. J., Grenville-Briggs, L. J., Griffith, J., Grünwald, N. J., Horn, K., Horner, N. R., Hu, C.-H., Huitema, E., Jeong, D.-H., Jones, A. M. E., Jones, J. D. G., Jones, R. W., Karlsson, E. K., Kunjeti, S. G., Lamour, K., Liu, Z., Ma, L., Maclean, D., Chibucos, M. C., McDonald, H., McWalters, J., Meijer, H. J. G., Morgan, W., Morris, P. F., Munro, C. A., O’Neill, K., Ospina-Giraldo, M., Pinzón, A., Pritchard, L., Ramsahoye, B., Ren, Q., Restrepo, S., Roy, S., Sadanandom, A., Savidor, A., Schornack, S., Schwartz, D. C., Schumann, U. D., Schwessinger, B., Seyer, L., Sharpe, T., Silvar, C., Song, J., Studholme, D. J., Sykes, S., Thines, M., van de Vondervoort, P. J., Phuntumart, V., Wawra, S., Weide, R., Win, J., Young, C., Zhou, S., Fry, W., Meyers, B. C., van West, P., Ristaino, J., Govers, F., Birch, P. R., Whisson, S. C., Judelson, H. S., and Nusbaum, C. 2009. Genome sequence and analysis of the Irish potato famine pathogen Phytophthora infestans. Nature 461:393-398.

Haverkort, A. J., Boonekamp, P. M., Hutten, R., Jacobsen, E., Lotz, L. A. P., Kessel, G. J. T., Visser, R. G. F., and Van der Vossen, E. A. G. 2008. Societal costs of late blight in potato and prospects of durable resistance through cisgene modification. Potato Res. 51:47-57.

James, W. C. 1971. An illustrated series of assessment keys for plant diseases, their preparation and usage. Can. Plant Dis. Surv. 51:39-65.

Jinks, J. L., and Grindle, M. 1963. Changes induced by training Phytophthora infestans. Heredity 18:245-264.

Judelson, H. S., and Roberts, S. 2002. Novel protein kinase induced during sporangial cleavage in the oomycete Phytophthora infestans. Eukaryot. Cell 1:687-695.

Kamoun, S., Furzer, O., Jones, J. D. G., Judelson, H. S., Ali, G. S., Dalio, R. J. D., Roy, S. G., Schena, L., Zambounis, A., Panabières, F., Cahill, D., Ruocco, M., Figueiredo, A., Chen, X.-R., Hulvey, J., Stam, R., Lamour, K., Gijzen, M., Tyler, B. M., Grünwald, N. J., Mukhtar, M. S., Tomé, D. F. A., Tör, M., Van Den Ackerveken, G., McDowell, J., Daayf, F., Fry, W. E., Lindqvist-Kreuze, H., Meijer, H. J. G., Petre, B., Ristaino, J., Yoshida, K., Birch, P. R. J., and Govers, F. 2015. The top 10 oomycete pathogens in molecular plant pathology. Mol. Plant Pathol. 16:413-434.

Kamoun, S., van West, P., de Jong, A. J., de Groot, K. E., Vleeshouwers, V. G. A. A., and Govers, F. 1997. A gene encoding a protein elicitor of Phytophthora infestans is down-regulated during infection of potato. Mol. Plant-Microbe Interact. 10:13-20.

Kasuga, T., Kozanitas, M., Bui, M., Huberli, D., Rizzo, D. M., and Garbelotto, M. 2012. Phenotypic diversification is associated with hostinduced transposon derepression in the sudden oak death pathogen, Phytophthora ramorum. PloS One 7:e34728.

Kim, D., Langemead, B., and Salzberg, S. L. 2015. HISAT: A fast spliced aligner with low memory requirements. Nat. Methods 12:357-360.

King, J. E., Colhoun, J., and Butler, R. D. 1968. Changes in the ultrastructure of sporangia of Phytophthora infestans associated with indirect germination and aging. Trans. Br. Mycol. Soc. 51:269-281.

Kirk, W. W., Abu-El Samen, F., Tumbalam, P., Wharton, P., Douches, D., Thill, C. A., and Thompson, A. 2009. Impact of different US genotypes of Phytophthora infestans on potato seed tuber rot and plant emergence in a range of cultivars and advanced breeding lines. Potato Res. 52:121-140.

Langmead, B., Trapnell, C., Pop, M., and Salzberg, S. L. 2009. Ultrafast and memory-efficient alignment of short DNA sequences to the human genome. Genome Biol. 10:R25.

Liu, D., Kashchandra, G. R., Hasegawa, P. M. and Bressan, R. A. 1994. Osmotin overexpression in potato delays development of disease symptoms. Proc. Nat'1. Acad. Sci. U.S.A. 91:1888-1892.

Manosalva, P. M., Park, S.-W., Forouhar, F., Tong, L., Fry, W. E., and Klessig, D. F. 2010. Methyl esterase 1 (StMES1) is required for systemic acquired resistance in potato. Mol. Plant-Microbe Interact. 23: 1151-1163.
Mayton, H., Griffiths, H., Simko, I., Cheng, S., Lorenzen, J., De Jong, W., and Fry, W. E. 2010. Foliar and tuber late blight resistance in a Solanum tuberosum breeding population. Plant Breed. 129:197-201.

McLeod, A., Fry, B. A., Zuluaga, A. P., Myers, K. L., and Fry, W. E. 2008. Toward improvements of oomycete transformation protocols. J. Eukaryot. Microbiol. 55:103-109.

Melhus, I. E. 1915. Germination and infection with the fungus of the late blight of potato (Phytophthora infestans). Research Bulletin 37. Agricultural Experiment Station. University of Wisconsin, Madison, WI, U.S.A. 64 pp.

Mizubuti, E. S. G., Aylor, D. E., and Fry, W. E. 2000. Survival of Phytophthora infestans sporangia exposed to solar radiation. Phytopathology 90:78-84.

Pfaffl, M. W. 2004. Quantification strategies in real-time PCR. Pages 87112 in: A-Z of Quantitative PCR, S.A. Bustin, ed. International University Line (IUL), La Jolla, CA, U.S.A.

Quast, C., Pruesse, E., Yilmaz, P., Gerken, J., Schweer, T., Yarza, P., Peplies, J., and Glockner, F. O. 2013. The SILVA ribosomal RNA gene database project: improved data processing and web-based tools. Nucl. Acids Res. 41:D590-D596.

Robinson, M. D., McCarthy, D. J. and Smyth, G. K. 2010. edgeR: A bioconductor package for differential expression analysis of digital gene expression data. Bioinformatics 26:139-140.

Small, I. M., Joseph, L., and Fry, W. E. 2015. Evaluation of the BlightPro decision support system for management of potato late blight using computer simulation and field validation. Phytopathology 105: 1545-1554.

Tani, S., and Judelson, H. 2006. Activation of zoosporogenesis-specific genes in Phytophthora infestans involves a 7-nucleotide promoter motif and cold-induced membrane rigidity. Eukaryot. Cell 5:745-752.

Tani, S., Kim, K. S., and Judelson, H. S. 2005. A cluster of NIF transcriptional regulators with divergent patterns of spore-specific expression in Phytophthora infestans. Fungal Genet. Biol. 42:42-50.

Tani, S., Yatzkan, E., and Judelson, H. S. 2004. Multiple pathways regulate the induction of genes during zoosporogenesis in Phytophthora infestans. Mol. Plant-Microbe Interact. 17:330-337.

Tian, Y., Yin, J., Sun, J., Ma, H., Ma, Y., Quan, J., and Shan, W. 2015. Population structure of the late blight pathogen Phytophthora infestans in a potato germplasm nursery in two consecutive years. Phytopathology 105:771-777.

Tokunaga, J., and Bartnicki-Garcia, S. 1971. Cyst wall formation and endogenous carbohydrate utilization during synchronous encystment of Phytophthora palmivora zoospores. Arch. Mikrobiol. 79:283-292.

Tooley, P. W., and Fry, W. E. 1985. Field assessments of fitness of isolates of Phytophthora infestans. Phytopathology 75:982-988.

Vargas, A. M., Quesada Ocampo, L. M., Céspedes, M. C., Carreño, N., González, A., Rojas, A., Zuluaga, A. P., Myers, K., Fry, W. E., Jiménez, P., Bernal, A. J., and Restrepo, S. 2009. Characterization of Phytophthora infestans populations in Colombia: First report of the A2 mating type. Phytopathology 99:82-88.

Vleeshouwers, V. G. A. A. and Oliver, R. P. 2013. Effectors as tools in disease resistance breeding against biotrophic, hemibiotrophic, and necrotrophic plant pathogens. Mol. Plant-Microbe Interact.. 27:196-206.

Zuluaga, A. P., Vega-Arreguín, J. C., Fei, Z., Ponnala, L., Lee, S. J., Matas, A. J., Patev, S., Fry, W. E., and Rose, J. K. C. 2016. Transcriptional dynamics of Phytophthora infestans during sequential stages of hemibiotrophic infection of tomato. Mol. Plant Pathol. 17:29-41.

\section{AUTHOR-RECOMMENDED INTERNET RESOURCE}

EnsembleProtists website:

http://protists.ensembl.org/Phytophthora_infestans/Info/Index 